\title{
Universal SNP Genotyping Assay with Fluorescence Polarization Detection
}

BioTechniques 31:560-570(September 2001)

\author{
T.M. Hsu, X. Chen ${ }^{1}$, S. Duan, \\ R.D. Miller, and P.-Y. Kwok \\ Washington University School \\ of Medicine, St. Louis, MO, and \\ ${ }^{1}$ Virginia Commonwealth Uni- \\ versity, Richmond, VA, USA
}

\section{ABSTRACT}

The degree of fluorescence polarization $(F P)$ of a fluorescent molecule is a reflection of its molecular weight $\left(\mathrm{M}_{\mathrm{r}}\right)$. FP is therefore a useful detection method for homogeneous assays in which the starting reagents and products differ significantly in $\mathrm{M}_{r}$. We have previously shown that FP is a good detection method for the single-base extension and the 5'-nuclease assays. In this report, we describe a universal, optimized single-base extension assay for genotyping single nucleotide polymorphisms (SNPS). This assay, which we named the templatedirected dye-terminator incorporation assay with fluorescence polarization detection (FP-TDI), uses four spectrally distinct dye terminators to achieve universal assay conditions. Even without optimization, approximately $70 \%$ of all SNP markers tested yielded robust assays. The addition of an $\mathrm{E}$. coli ssDNA-binding protein just before the FP reading significantly increased $F P$ values of the products and brought the success rate of FP-TDI assays up to 90\%. Increasing the amount of dye terminators and reducing the number of thermal cycles in the single-base extension step of the assay increased the separation of the FP values between the products corresponding to different genotypes and improved the success rate of the assay to $100 \%$. In this study, the genomic DNA samples of 90 individuals were typed for a total of 38 FP-TDI assays (using both the sense and antisense TDI primers for 19 SNP markers). With the previously described modifications, the FP-TDI assay gave unambiguous genotyping data for all the samples tested in the 38 FP-TDI assays. When the genotypes determined by the FPTDI and 5'-nuclease assays were compared, they were in $100 \%$ concordance for all experiments (a total of 3420 genotypes). The four-dye-terminator master mixture described here can be used for assaying any SNP marker and greatly simplifies the SNP genotyping assay design.

\section{INTRODUCTION}

Simple, robust, and flexible genotyping assays for single nucleotide polymorphisms (SNPs) that can be designed quickly and implemented without much optimization are highly desirable in the fine mapping of genetic factors associated with complex disease traits. In these instances, a candidate region is scanned for SNPs, which are then used in various methods to narrow the candidate region further. In these end-game situations, one cannot afford to wait for specialty probes to be made or lengthy optimization steps to be taken before genotyping all the SNPs found in the candidate regions.

Because the fluorescence polarization (FP) value of a fluorescent molecule is a reflection of its molecular weight $\left(M_{\mathrm{r}}\right)$, FP is a useful detection method for homogeneous assays in which the starting reagents and products differ significantly in size. We have previously shown that FP is a good detection method for the singlebase extension and the $5^{\prime}$-nuclease assays $(5,11)$. Here, we describe a universal, optimized, single-base extension assay for genotyping SNPs. This FPTDI assay uses four spectrally distinct dye terminators to achieve universal assay conditions for the most abundant type of DNA sequence variation in the human genome. The ability to genotype these SNPs rapidly in a cost-effective fashion is becoming increasingly important in clinical genetic testing, forensic medicine, agricultural biotechnology, population genetics, and the new field of pharmacogenomics (10). Several high-throughput methods currently in use for typing SNPs [including the high-density chip arrays for allele-specific hybridization analysis $(22,24)$, the homogeneous $5^{\prime}$-nuclease (TaqMan ${ }^{\circledR}$ ) assay (12), the dye-labeled oligonucleotide ligation (6), the fluorescent resonance energy tranfer (FRET)-TDI assay $(4,7)$, and the homogeneous molecular beacon allelespecific oligonucleotide assay (21)] all require specialty probes $(9,17)$. The FP-TDI assay, which is based on tem plate-directed primer extension, has several advantages over these other genotyping assays. The FP-TDI assay is more cost effective because it does not require modified probes such as dye-labeled primers. The FP-TDI assay also offers the flexibility of using the best markers as they become available without having to redesign dye-labeled probes or high-density DNA chips. Furthermore, the FP-TDI assay is easy to set up and is readily scalable for large-scale genetic studies.

Even though the original FP-TDI assay was a robust and homogeneous assay for SNP genotyping, it did not work consistently for all the markers that were tested. Without optimization, as many as $30 \%$ of TDI primers did not produce clear-cut genotyping data (ei- 


\section{Research Report}

ther the differences between clusters of different genotypes were too small or the data points were not tightly clustered). Here, we describe significant improvements that make the FP-TDI assay extremely robust and easy to optimize. We performed our study with 19 markers (38 TDI primers including both sense and antisense TDI primers for each marker) on DNA samples from 90 individuals. We demonstrate that the addition of $E$. coli ssDNA-binding (SSB) protein and reading buffer (containing glycerol and ethanol), together with minor adjustments in the thermal cycling conditions, improves FP analysis tremendously.

\section{MATERIALS AND METHODS}

\section{Materials}

DNA samples from 90 individuals in the National Institutes of Health (NIH) Polymorphism Discovery Panel (PD0001-PD0090) were used in this study. All reactions were run and read in 96-well black-skirted plates purchased from MJ Research (Waltham, MA, USA). Publicly available markers from the dbSNP database (http:// www.ncbi.nlm.nih.gov/SNP/) were used in this study. The dbSNP assay numbers are listed in Table 1. PCR and TDI primers are also listed in Table 1. All primers were obtained from Invitrogen (Carlsbad, CA, USA).

AmpliTaq Gold ${ }^{\circledR}$ and AmpliTaq ${ }^{\circledR}$ FS DNA polymerases were obtained from Applied Biosystems (Foster City, CA, USA). Shrimp alkaline phosphatase was purchased from Roche Molecular Biochemicals (Indianapolis, IN, USA), and E. coli exonuclease I was purchased from Amersham Pharmacia Biotech (Piscataway, NJ, USA). dNTPs labeled with Rox, Bodipy ${ }^{\circledR}$ Fluorescein, Tamra, and R6G were generous gifts from PerkinElmer (Boston, MA, USA), and SSB protein was purchased from USB (Cleveland, OH, USA).

\section{PCR Amplification}

Human genomic DNA (20 ng) was amplified in $10-\mu \mathrm{L}$ reaction mixtures containing $1 \mu \mathrm{L} 10 \times$ PCR buffer (Applied Biosystems), $1 \mu \mathrm{L} 25 \mathrm{mM} \mathrm{MgCl}_{2}$, $0.2 \mu \mathrm{L} 2.5 \mathrm{mM}$ dNTP, $0.5 \mu \mathrm{L}(2.5 \mu \mathrm{M}$ each) PCR primers, and $0.25 \mathrm{U}$ AmpliTaq Gold DNA polymerase. The reaction mixture was held at $95^{\circ} \mathrm{C}$ for 10 min for AmpliTaq Gold enzyme activation, followed by 35 amplification cy-

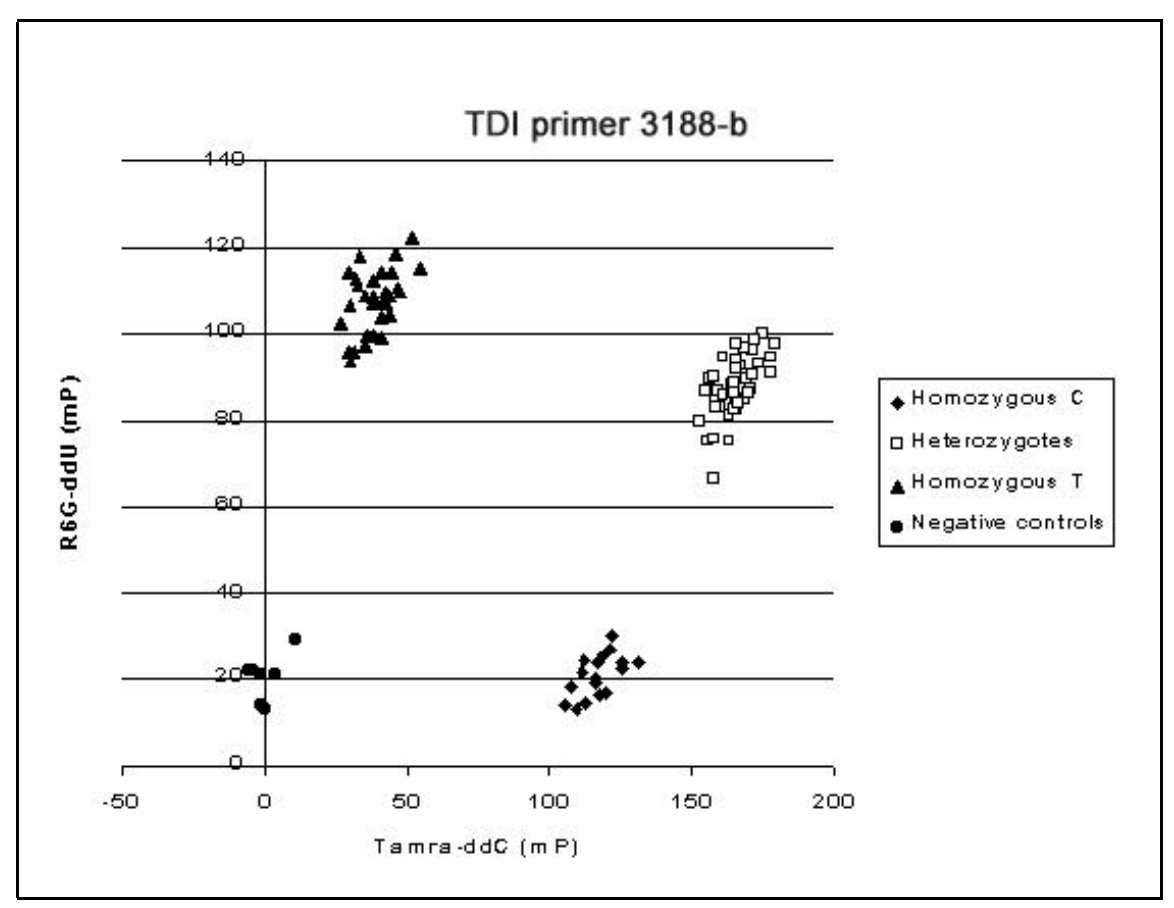

Figure 1. FP-TDI genotyping data for TDI primer 3188-b (antisense TDI primer for marker 3188).

cles. Each cycle consisted of denaturation at $93^{\circ} \mathrm{C}$ for $30 \mathrm{~s}$, primer annealing at $58^{\circ} \mathrm{C}$ for $40 \mathrm{~s}$, and primer extension at $72^{\circ} \mathrm{C}$ for $40 \mathrm{~s}$. The reaction mixtures were then incubated at $72^{\circ} \mathrm{C}$ for $5 \mathrm{~min}$ for final primer extension. At the end of the reaction, the reaction mixtures were held at $4^{\circ} \mathrm{C}$ until further use.

\section{Excess PCR Primer and dNTP Degradation}

At the end of the PCR assay, $10 \mu \mathrm{L}$ enzymatic cocktail were added to the PCR mixture. This enzymatic cocktail contained 1 U shrimp alkaline phosphatase, $1 \mathrm{U}$ E. coli exonuclease I, and $1 \mu \mathrm{L}$ shrimp alkaline phosphatase buffer (0.5 M Tris-HCl, pH 8.5, and 50 $\mathrm{mM} \mathrm{MgCl} 2$ ). The mixture was incubated at $37^{\circ} \mathrm{C}$ for $45 \mathrm{~min}$ before the enzymes were heat-inactivated at $95^{\circ} \mathrm{C}$ for $15 \mathrm{~min}$. The reaction mixture was kept at $4^{\circ} \mathrm{C}$ until further use.

\section{TDI Primer Design}

Primer Express ${ }^{\mathrm{TM}}$ (Applied Biosystems) was used to design the TDI primers. The TDI primers were designed to have melting temperatures between $60^{\circ} \mathrm{C}$ and $80^{\circ} \mathrm{C}$ and lengths between 20 and 30 bases (about $10000 \mathrm{Da}$ ).

\section{Single-Base Extension}

To the enzymatically treated PCR product, we added $10 \mu \mathrm{L}$ TDI cocktail containing $2 \mu \mathrm{L} 5 \times$ TDI buffer (250 $\mathrm{mM}$ Tris- $\mathrm{HCl}, \mathrm{pH} 9.0,250 \mathrm{mM} \mathrm{KCl}$, $25 \mathrm{mM} \mathrm{NaCl}, 25 \mathrm{mM} \mathrm{MgCl} 2$, and $40 \%$ glycerol), $1 \mu \mathrm{L} 10 \mu \mathrm{M}$ TDI primer, 0.4 $\mathrm{U}$ Thermo Sequenase ${ }^{\mathrm{TM}}$ (Amersham Pharmacia Biotech), and $0.05 \mu \mathrm{L}$ fourdye-ddNTP mixture ( $25 \mu \mathrm{M}$ each). The reaction mixture was incubated at $93^{\circ} \mathrm{C}$ for $1 \mathrm{~min}$, followed by 50 cycles of $93^{\circ} \mathrm{C}$ for $10 \mathrm{~s}$ and $50^{\circ} \mathrm{C}$ for $30 \mathrm{~s}$. At the end of the reaction, the samples were held at $4^{\circ} \mathrm{C}$.

\section{E. coli SSB Protein and Reading Buffer (Optional)}

After the primer extension reaction, $10 \mu \mathrm{L}$ of a mixture containing $0.1 \mu \mathrm{L}$ $2.2 \mu \mathrm{g} / \mu \mathrm{L}$ SSB protein, $2 \mu \mathrm{L} 5 \times$ TDI buffer, and $7.9 \mu \mathrm{L}$ double-distilled water were added. The reaction mixtures 


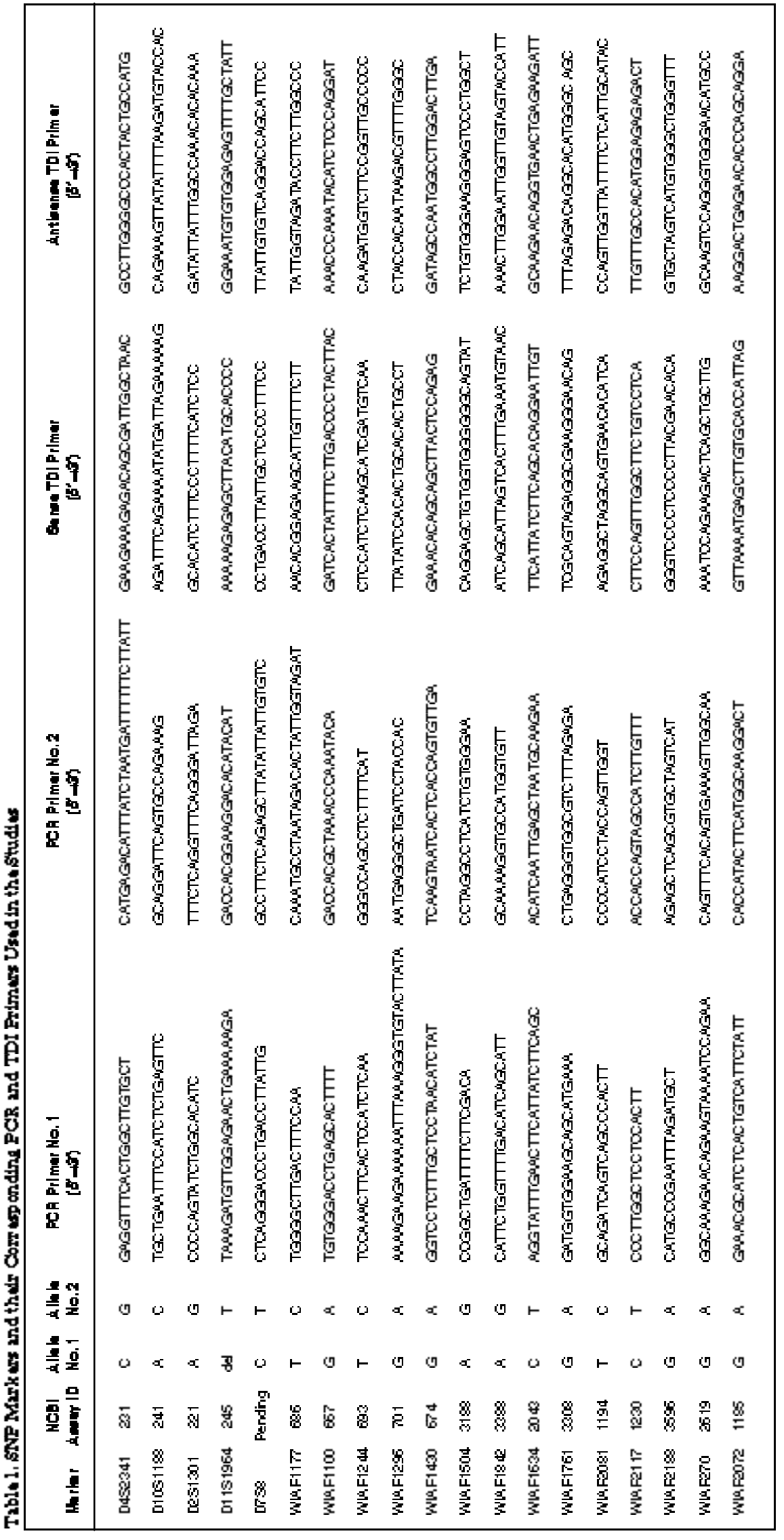

were incubated at $37^{\circ} \mathrm{C}$ for $1 \mathrm{~h}$ and then held at $4^{\circ} \mathrm{C}$. After SSB protein incubation, $75 \mu \mathrm{L}$ reading buffer (consisting of $25 \mu \mathrm{L} 100 \%$ ethanol, $10 \mu \mathrm{L} 5 \times$ TDI buffer, and $40 \mu \mathrm{L}$ double-distilled water) were added to each well.

\section{FP Measurement}

FP measurement was done on an LJL Analyst ${ }^{\mathrm{TM}}$ fluorescence reader (Molecular Devices, Sunnyvale, CA, USA). FP value is defined by the formula:

$$
P=(I v v-I v h) /(I v v+I v h)
$$

[Eq. 1]

where $I v v$ is the emission intensity measured when the excitation and emission polarized filters are parallel and $I v h$ is the emission intensity measured when these filters are perpendicular $(1,23)$. Polarization is closely related to anisotropy, which is an alternative system relating these measurements $(23,24)$. In essence, polarization is twice the fraction of light that is polarized. It is useful to express the measurements as millipolarization $(\mathrm{mP})$ :

$$
\begin{aligned}
& m P=1000 \times(I v v-(G \times I v h)) / \\
& (I v v+(G \times I v h))
\end{aligned}
$$

where $G$ is a gating factor near the value of one used to adjust for slight differences in the two optical paths used (according to the manufacturer's information). $G$ was estimated for each of the four dyes using an assumed $m P$ value for the dye of $30 \mathrm{mP}$ and measured values for $I v v$ and $I v h$.

\section{Corrections for Spectral Overlaps}

The observed and true intensity values for $n$ different filter sets can be respectively represented as $(n \times 1)$ vectors $\mathrm{C}$ and $\mathrm{B}$, and

$$
\mathrm{AB}=\mathrm{C}
$$

where $\mathrm{A}$ is an $(n \times n)$ matrix. A can be experimentally calculated by measuring the fraction of intensity of a single dye (column) transmitted through each of the filter sets (rows). By left matrix multiplication, it can be shown that

$$
\mathrm{A}^{-1} \mathrm{C}=\mathrm{B}
$$

where $A^{-1}$ is the determinant of $A$. The true intensity value, $B$, can then be calculated. 


\section{RESULTS}

Nineteen published SNP markers were used in this study (Table 1). Genomic DNA from each of 90 individuals in the NIH Discovery Panel was typed for each marker, and six samples with no DNA served as negative controls. Both the sense and the antisense TDI primers were typed for each marker for a total of 38 TDI experiments (3420 genotypes). For each TDI experiment, we used a four-dye-ddNTP mixture consisting of ROX-ddGTP, Bodipy-Fluorescein-ddATP, TAMRAddCTP, and R6G-ddUTP (Table 2).

Following a standard protocol, the FP readings of the samples clustered into four distinct groups without optimization for 27 out of 38 TDI primers (71\% success rate). Figure 1 shows the results of a typical assay. As expected, the negative controls without DNA have low FP values for both dyes analyzed and occupy the area near the origin of the plot, indicating that the small dye terminators remain free in solution. For homozygous $\mathrm{C}$ individuals, the FP values for TAMRA-ddC are high and the values for R6G-ddU are low, reflecting significant incorporation of the TAMRA-ddC terminator onto the TDI primer but minimal incorporation of the R6G-ddU. Conversely, the FP values of TAMRAddC are low and those of R6G-ddU are high for homozygous $\mathrm{T}$ individuals. For heterozygous individuals, the FP values for both TAMRA-ddC and R6G-ddU are high because of significant incorporation of both dye terminators.

Without optimization, the FP values did not separate into four distinct clusters for 11 out of 38 FP-TDI assays. Scatter plots of two examples (assays 245-b and 674-a) are shown in Figure 2 , A and B. Although hints of clusters were seen, the separations were not clear-cut enough to call genotypes unambiguously. To increase the separations between the clusters, two additional steps were added to the protocol. The first step involved the incubation of each reaction mixture with $E$. coli SSB protein for $1 \mathrm{~h}$ after the completion of the single-base extension reaction. In the second step, a reading buffer containing ethanol and glycerol was added to each reaction mixture after the com pletion of SSB protein incubation. With the addition of these two steps, all 11 primers showed improvement (Figure 2, C and D); however, four out of 11 FP-TDI assays did not produce distinct enough clusters.

In the four remaining assays, the homozygotes for one allele were clearly separated from the controls and the samples of other genotypes, but the homozygotes of the other allele and the heterozygotes could not be distinguished clearly (Figure 3, A and B). We reasoned that the merging of the homoand heterozygotes in these cases was due to the misincorporation of dye terminators when the terminator for the correct allele was used up prematurely. Accordingly, we added more dye terminators to the reaction and reduced the number of cycles used in the singlebase extension reaction. With these minor modifications, all four assays showed dramatic improvement with easily distinguishable clusters of FP values (Figure 3, C and D).

Because a four-dye-ddNTP mixture was used, there were some spectral overlaps between certain dye-ddNTPs. Even though these spectral overlaps did not affect our data significantly, we decided to investigate the degree of spectral overlaps and devise ways to correct for them. Using each of the four fluorescent ddNTPs and our filter set, we investigated the overlap in spectral detection. When the six pairs of dyes were considered, little or no overlap was detected with BODIPY-ddA and TAMRA-ddC, BODIPY-ddA and ROX-ddG, and ROX-ddG and R6G-ddU. However, some fluorescent overlap was detected with the other three combinations, with the highest amounts being 16\% (BOD IPY-ddA with R6G filters), 11\% (R6GddU with the TAMRA filters), 9\% (ROX-ddG with TAMRA filters), and 7\% (TAMRA-ddC with R6G filters). For some assays, calculations of FP values using corrections for spectral overlaps gave enhanced separation of groups in scatter plots (Figure 4, A and B).

The genotypes called based on FPTDI assay and $5^{\prime}$-nuclease assay were compared for all 38 experiments (11). The concordance rate of these experiments is $100 \%$ for all markers after 3420 genotypes. The genotyping data have been deposited into the public database, dbSNP. 


\section{DISCUSSION}

For association studies using SNP markers, many new genotyping assays must be developed quickly for testing hundreds of samples. Therefore, ease of optimization and assay implementation is very important. Although the original protocol for the FP-TDI assay worked quite well, it was optimized for two dye terminators (ROX and TAMRA). A different reagent mixture must be assembled for each marker when it is being typed. In this report, we describe a universal master mixture for the assay that is applicable for all SNP markers. In addition, we outline a very simple assay development strategy for robust assays.

We identified a set of four spectrally distinct dye terminators for this assay and have incorporated an analysis algorithm that removed the spectral overlaps between these dye terminators. This set of dye terminators greatly simplifies assay design. As we were developing the four-color FP-TDI assay, we encountered a number of failures. The careful examination of the results of the failed assays allowed us to determine that there were three sources of problems in these assays: $(i)$ the single-base extension reaction is not pushed to completion, (ii) AmpliTaq FS incorporates dye terminators nonspecifically when the correct dye terminator is not available, and (iii) the FP difference between primer extension product and unincorporated dye terminator is too small.

As a homogeneous assay, the FP reading at the end of the assay is a com posite value, reflecting the total sum of free and incorporated dye terminators. When all dye terminators are incorporated, the full FP difference between the primer extension product and the free dye terminator is realized. If the reaction is incomplete, only a fraction of the theoretical FP difference is observed and the result is sub-optimal. On the other hand, if the reaction was pushed beyond completion (i.e., one of the dye terminators was depleted during an early part of the reaction), then AmpliTaqFS may incorporate other dye terminators nonspecifically in place of the unavailable correct dye terminator (16). The degree of misincorporation is sequence-dependent. Rox-ddG is misincorporated most frequently, but Bod-
Table 2. The Excitation Wavelengths and Emission Wavelengths of the Dye Terminators

\begin{tabular}{|lcc|}
\hline Dye-terminators & Excitation Wavelength & Emission Wavelength \\
\hline Rox-ddGTP & $580 \mathrm{~nm}$ & $605 \mathrm{~nm}$ \\
Bodipy FI-14-ddATP & $501 \mathrm{~nm}$ & $510 \mathrm{~nm}$ \\
Tamra-ddCTP & $552 \mathrm{~nm}$ & $575 \mathrm{~nm}$ \\
R6G-ddUTP & $525 \mathrm{~nm}$ & $550 \mathrm{~nm}$ \\
& & \\
\hline
\end{tabular}

ipy-ddA is rarely misincorporated. We have not observed any problems with misincorporation for either Tamra-ddC or R6G-ddU.

To correct the problems of incom plete reactions and misincorporation of dye terminators, we adjusted the number of thermal cycles and the amount of dye terminators in the single-base extension step to optimize genotyping data. Deciding whether to adjust each of these factors upward or downward depends on individual FP-TDI assays. When the FP values of the heterozygous samples are low compared to those of the homozygous samples, the single-base extension reaction has not been pushed to completion. To achieve better results, two adjustments can be made: (i) increase the number of thermal cycles and (ii) decrease the number of dye terminators. These two changes have the effect of pushing the reaction closer to completion and therefore increase the FP difference between hetero- and homozygous samples. However, if the reaction was pushed to completion too early, then the opposite effects can occur. When one of the dye terminators is used up early in the reac-

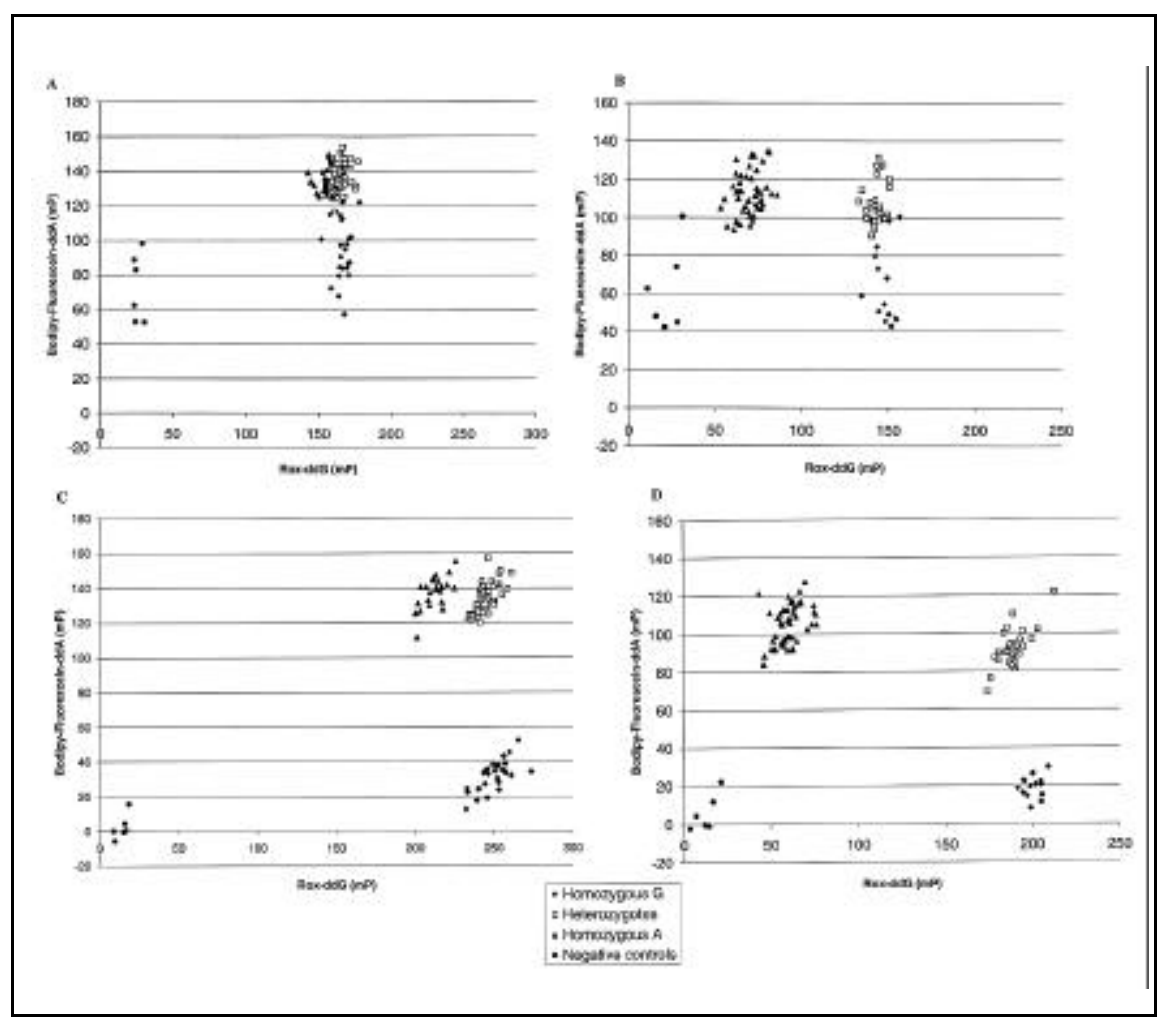

Figure 2. FP-TDI genotyping data for TDI primer 245-b and TDI primer 674-a. (A) FP-TDI results for TDI primer 245-b without the addition of SSB protein or reading buffer. (B) FP-TDI results for TDI primer 674-a without the addition of SSB protein or reading buffer. (C) FP-TDI results for TDI primer 245-b with the addition of SSB protein and reading buffer. (D) FP-TDI results for TDI primer 674-a with the addition of SSB protein and reading buffer. 
tion, the AmpliTaq-FS enzyme may incorporate the wrong dye terminator in place of the depleted correct dye terminator. As a result, the FP values of the homozygous samples may be too high, and they frequently merge with those of the heterozygous samples. By increasing the amount of dye terminators and decreasing the number of thermal cycles, we showed that this problem could be remedied.

Our data also showed that the TDI

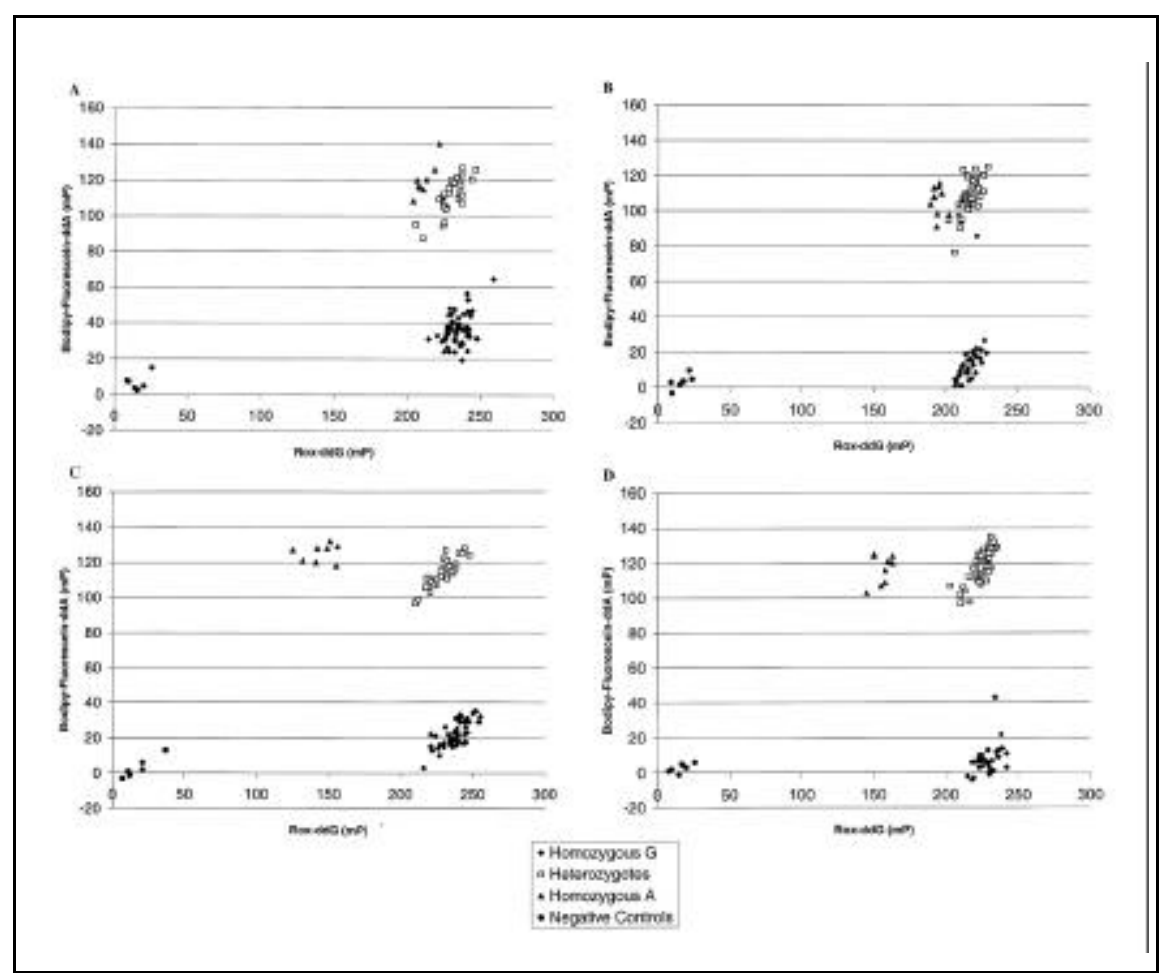

Figure 3. FP-TDI genotyping data for TDI primers 3388-a and 693-b. (A) FP-TDI results for TDI primer 3388-a with 50 thermal cycles and 2.5 pmol dye terminators in the single-base extension step. (B) FP-TDI results for TDI primer 693-b with 50 thermal cycles and 2.5 pmol dye terminators in the singlebase extension step. (C) FP-TDI results for TDI primer 3388-a with 25 thermal cycles and 5.0 pmol dye terminators in the single-base extension step. (D) FP-TDI results for TDI primer 693-b with 25 thermal cycles and $5.0 \mathrm{pmol}$ dye terminators in the single-base extension step.

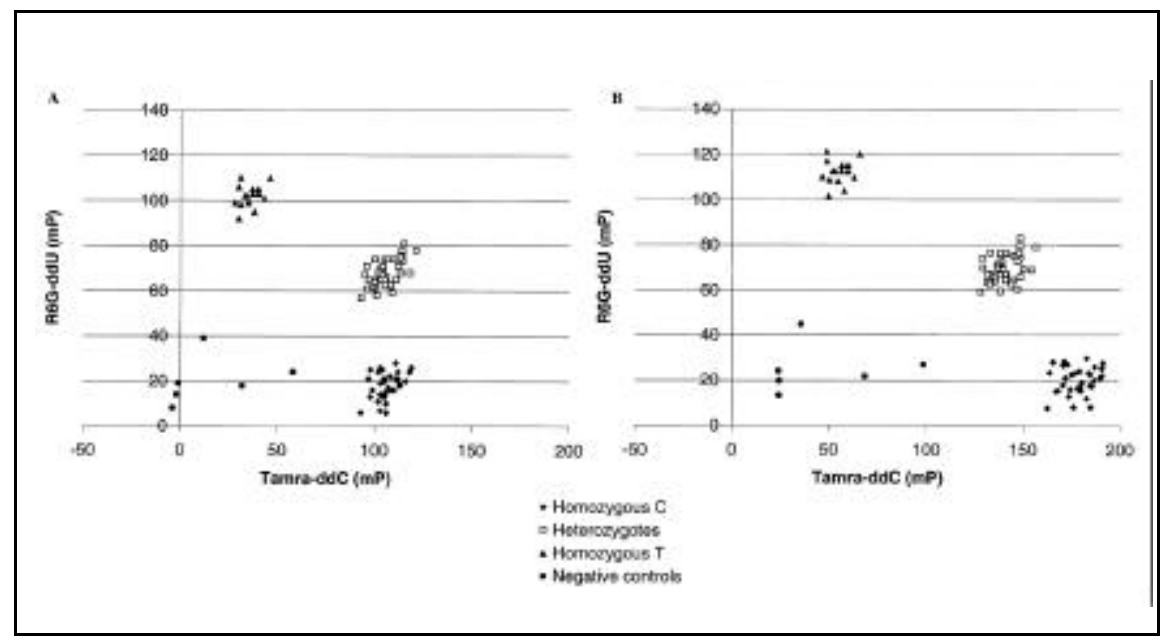

Figure 4. FP-TDI genotyping data for TDI primer 3308-b without the addition of SSB protein or reading buffer. (A) Results taken directly from the LJL Analyst plate reader with no correction for spectral overlap. (B) The same results applying a correction for spectral overlap. 


\section{Research Report}

assay improved significantly with the addition of the $E$. coli SSB protein. With SSB protein, the FP difference between the positive reaction (primer extension products) and the negative reaction (unincorporated dye terminators) was increased for all TDI primers. This increased FP difference can be explained by the properties of the $E$. coli SSB protein. E. coli SSB was first identified and purified by Sigal et al. (20), and it binds preferentially to ssDNA. E. coli SSB protein is unique among SSB proteins in that it forms a stable homotetramer in solution $(3,13,23)$. This SSB tetramer binds to ssDNA in different modes depending on the salt concentration and SSB protein density $(1,8,14,15)$. Our single-base extension reaction has a high salt concentration and low SSB protein density. Under these conditions, the SSB tetramers bind to ssDNA in the

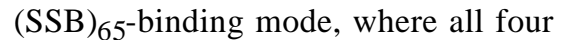
subunits interact with ssDNA $(1,2,14$,
18). Our TDI primers have an average size of between 24 and 28 bp and correspond to an $M_{\mathrm{r}}$ of about $10000 \mathrm{Da}$ (1). Each SSB protein has an $M_{\mathrm{r}}$ of about $18800 \mathrm{Da}$, and a tetramer has an $M_{\mathrm{r}}$ of about $75200 \mathrm{Da}$ (19). Therefore, the $M_{\mathrm{r}}$ of the ssDNA-SSB tetramer complex is about $85200 \mathrm{Da}$. This increase in $M_{\mathrm{r}}$ leads to an increase in the FP value of the primer extension product. As a result, the FP difference between positive and negative reactions increases. In addition to increasing the $M_{\mathrm{r}}$ of the primer extension products, the SSB tetramers may also enhance FP via their disruption of the secondary structures of these primer extension products. This disruption of the secondary structures helps to extend these primer extension products into open nucleoprotein fiber loops, which have a greater molecular volume than that of collapsed ssDNA without SSB protein (8).

Because of possible spectral over- laps between various dye terminators, previous papers have used a master mixture with only two fluorescent dye terminators in each reaction. This report shows that despite some degree of spectral overlaps, the modified FP-TDI assay is so robust that using a master mixture of four fluorescent dye terminators still gives clear-cut genotyping data for all 38 FP-TDI assays. We also investigated the extent of spectral overlaps and have come up with calculations to correct for them in case one needs to subtract these overlaps to obtain clear-cut genotyping data (see Materials and Methods). Even though using a master mixture of four-dye terminators is slightly more expensive than using a master mixture of two-dye terminators, we think that using a fourdye master mixture has two major advantages: $(i)$ it simplifies genotyping protocols and reduces human errors and (ii) it allows for multiplexing (us- 
ing a four-dye master mixture allows one to perform the FP-TDI assay for two markers simultaneously in one reaction as long as the two markers require non-overlapping pairs of fluorescent dye terminators).

Although the FP-TDI assay is one of several different methods that are currently available for automated genotyping, it has a number of advantages. First, this assay is less costly to develop because it does not require any modified probes. The two PCR primers and one TDI probe can be obtained quickly and very inexpensively. Second, as shown in this report, single-base incorporation assays are very easy to optimize. One can literally develop a robust assay within hours of obtaining the primers. No redesigning or lengthy manufacturing of specialty probes or microarrays is necessary when a new marker is needed for a study. Finally, FP-TDI assay uses FP as a detection format, which is independent of fluorescence intensity and requires no separation of free from bound dye ddNTP. As the number of SNPs available for typing dramatically increases as a result of several SNP discovery efforts, flexible and robust genotyping assays such as the four-color FP-TDI assay described in this report will be of great utility.

\section{ACKNOWLEDGMENTS}

We thank S. Spurgeon (Applied Biosystems), P. Buzby (PerkinElmer), and R. Greene (PerkinElmer) for generous gifts of reagents. This work is supported by the National Institutes of Health grant no. R01-EY12557 to P.-Y.K. and grant no. T32-AR07284 to T.M.H.

\section{REFERENCES}

1.Bujalowski, W. and T.M. Lohman. 1986. Escherichia coli single-strand binding protein forms multiple, distinct complexes with single-stranded DNA. Biochemistry 25:77997802.

2.Bujalowski, W. and T.M. Lohman. 1989. Negative co-operativity in Escherichia coli single strand binding protein-oligonucleotide interactions. II. Salt, temperature and oligonucleotide length effects. J. Mol. Biol. 207:269288.

3.Chase, J.W. and K.R. Williams. 1986. Single-stranded DNA binding proteins required for DNA replication. Annu. Rev. Biochem.
55:103-136.

4.Chen, X. and P.-Y. Kwok. 1997. Template-directed dye-terminator incorporation (TDI) assay: a homogeneous DNA diagnostic method based on fluorescence energy transfer. Nucleic Acids Res. 25:347-353.

5.Chen, X., L. Levine, and P.-Y. Kwok. 1999. Fluorescence polarization in homogeneous nucleic acid analysis. Genome Res. 9:492498.

6.Chen, X., K.J. Livak, and P.-Y. Kwok. 1998. A homogeneous, ligase-mediated DNA diagnostic test. Genome Res. 8:549-556.

7.Chen, X., B. Zehnbauer, A. Gnirke, and P.Y. Kwok. 1997. Fluorescence energy transfer detection as a homogeneous DNA diagnostic method. Proc. Natl. Acad. Sci. USA 94:10756-10761.

8.Chrysogelos, S. and J. Griffith. 1982. Escherichia coli single-strand binding protein organizes single-stranded DNA in nucleosome-like units. Proc. Natl. Acad. Sci. USA 79:5803-5807.

9.Hertzberg, R.P. and A.J. Pope. 2000. Highthroughput screening: new technology for the 21 st century. Curr. Opin. Chem. Biol. 4:445451 .

10.Kwok, P.-Y. and Z. Gu. 2000. Single nucleotide polymorphism (SNP): genetic marker of the new millennium? Gene Med. 4:37-43.

11.Latif, S., I. Bauer-Sardina, K. Ranade, K.J. Livak, and P.-Y. Kwok. 2001. Fluorescence polarization in homogeneous nucleic acid analysis II: 5'-nuclease assay. Genome Res. $11: 436-440$

12.Livak, K.J., J. Marmaro, and J.A. Todd. 1995. Towards fully automated genome-wide polymorphism screening. Nat. Genet. 9:341342.

13.Lohman, T.M. and M.E. Ferrari. 1994. Escherichia coli single-stranded DNA-binding protein: multiple DNA-binding modes and cooperativities. Annu. Rev. Biochem. 63:527570.

14.Lohman, T.M. and L.B. Overman. 1985. Two binding modes in Escherichia coli single strand binding protein-single stranded DNA complexes. Modulation by $\mathrm{NaCl}$ concentration. J. Biol. Chem. 260:3594-3603.

15.Lohman, T.M., L.B. Overman, and S. Datta. 1986. Salt-dependent changes in the DNA binding co-operativity of Escherichia coli single strand binding protein. J. Mol. Biol. 187:603-615.

16.Parker, L.T., H. Zakeri, Q. Deng, S. Spurgeon, P.-Y. Kwok, and D.A. Nickerson. 1996. AmpliTaq DNA polymerase, FS dyeterminator sequencing: analysis of peak height patterns. BioTechniques 21:694-699.

17.Pope, A.J., U.M. Haupts, and K.J. Moore. 1999. Homogeneous fluorescence readouts for miniaturized high-throughput screening: theory and practice. Drug Discov. Today 4:350-362.

18.Raghunathan, S., A.G. Kozlov, T.M. Lohman, and G. Waksman. 2000. Structure of the DNA binding domain of $E$. coli S B protein bound to ssDNA. Nat. Struct. Biol. 7:648-652.

19.Sancar, A., K.R. Williams, J.W. Chase, and W.D. Rupp. 1981. Sequences of the ssb gene and protein. Proc. Natl. Acad. Sci. USA
78:4274-4278.

20.Sigal, N., H. Delius, T. Kornberg, M.L. Gefter, and B. Alberts. 1972. A DNA-unwinding protein isolated from Escherichia coli: its interaction with DNA and with DNA polymerases. Proc. Natl. Acad. Sci. USA 69:3537-3541.

21.Tyagi, S., D.P. Bratu, and F.R. Kramer. 1998. Multicolor molecular beacons for allele discrimination. Nat. Biotechnol. 16:49-53.

22.Wang, D.G., J.B. Fan, C.J. Siao, A. Berno, P. Young, R. Sapolsky, G. Ghandow, N. Perkins et al. 1998. Large-scale identification, mapping, and genotyping of single-nucleotide polymorphisms in the human genome. Science 280:1077-1082.

23.Wold, M.S. 1997. Replication protein A: a heterotrimeric, single-stranded DNA-binding protein required for eukaryotic DNA metabolism. Annu. Rev. Biochem. 66:61-92.

24.Yershov, G., V. Barsky, A. Belgovskiy, E. Kirillov, E. Kreindlin, I. Ivanov, S. Parinov, D. Guschin et al. 1996. DNA analysis and diagnostics on oligonucleotide microchips. Proc. Natl. Acad. Sci. USA 93:4913-4918.

Received 21 December; accepted 6 April 2001.

Address correspondence to:

Dr. Pui-Yan Kwok

Division of Dermatology

Washington University

660 S. Euclid Avenue, P.O. Box 8123

St. Louis, MO 63110, USA

e-mail:kwok@genetics.wustl.edu

For reprints of this or any other article, contact Reprints@BioTechniques.com 